\title{
Ultrafast excited-state dynamics of strongly coupled porphyrin/core-substituted-naphthalenediimide dyads $\dagger$
}

\author{
Natalie Banerji, ${ }^{a}$ Sheshanath V. Bhosale, ${ }^{* b}$ Irina Petkova, ${ }^{a}$ Steven J. Langford ${ }^{b}$ \\ and Eric Vauthey*a
}

Received 19th August 2010, Accepted 4th October 2010

DOI: $10.1039 / \mathbf{c 0 c p 0 1 5 4 4 j}$

The photophysics and excited-state dynamics of two dyads consisting of either a free-base or a zinc-tetraphenylporphyrin linked through a rigid bridge to a core-substituted naphthalenediimide (NDI) have been investigated by femtosecond-resolved spectroscopy. The absorption and fluorescence spectra differ substantially from those of the individual units, pointing to a substantial coupling and to a delocalisation of the excitation over the whole molecule, as confirmed by quantum chemistry calculations. A strong dependence of their excited-state dynamics on the solvent polarity has been observed. In toluene, the fluorescence quantum yield of the dyads is of the order of a few percent and the main decay channel of the emitting state is proposed as intersystem-crossing to the triplet state. However, in a medium polarity solvent like dichloromethane, the emitting state undergoes charge separation from the porphyrin to the NDI unit within 1-3 ps, and the ensuing charge-separated state recombines in about 10-20 ps. This solvent dependence can be explained by the weak driving force for charge separation in polar solvents and the large electronic coupling between the porphyrin and NDI moieties, making charge separation a solvent-controlled adiabatic process.

\section{Introduction}

Over the past few years, impressive efforts have been invested in the development of multichromophoric organic molecules with specific properties for various applications, such as optoelectronics, photovoltaics or sensing to name just a few. ${ }^{1-8}$ The marriage of chemically different chromophoric pieces into a single molecular system results into a chemical entity either combining the properties of each unit or exhibiting novel features. The key parameter for this outcome is the coupling between the chromophores. Weak coupling is normally achieved by restraining electronic communication between the two moieties, keeping them far apart or orienting them in an orthogonal fashion with properly designed bridging units. Strong coupling results from a short interchromophoric distance and an adequate orientation and/or by the use of a conjugated bridging unit.

Porphyrins are probably the most used building blocks in multichromophoric systems. Their widespread popularity arises from their similarity to natural pigment systems as well as from the possibility to tune their optical and redox properties by varying the nature of the substituents and/or of the central

\footnotetext{
${ }^{a}$ Department of Physical Chemistry, University of Geneva,

CH-1211Geneva 4, Switzerland.E-mail: eric.vauthey@unige.ch

${ }^{b}$ School of Chemistry, Monash University, Clayton, Victoria-3800,

Australia.E-mail: sheshanath.bhosale@monash.edu,

Fax: + 61 399054597; Tel: +61399055980

$\dagger$ Electronic supplementary information (ESI) available: Synthesis of FbTPPNDI and ZnTPPNDI, table with absorption and emission maxima, transient absorption spectra measured with FbTPP in toluene upon 400 and $615 \mathrm{~nm}$ excitation, with ZnTPP upon $400 \mathrm{~nm}$ excitation, with FbTPPNDI in toluene upon $615 \mathrm{~nm}$ excitation at moderate and high concentration, and with FbTPPNDI in DCM upon $615 \mathrm{~nm}$ excitation. See DOI: 10.1039/c0cp01544j
}

metal atom. For example, free-base and $\mathrm{Zn}$-porphyrins can be combined to form arrays for light harvesting, ${ }^{9,10}$ whereas the association of free-base or zinc with gold porphyrins results to dyads for charge separation (CS). ${ }^{11,12}$ However, in most dyads with charge transfer properties, the porphyrin is both the chromophore and the electron donor, and the role of the electron-accepting group is ensured by a quinone or an equivalent unit with a high reduction potential and usually no or very low absorbance in the visible range. For example, the Marcus inverted region for intramolecular charge recombination and charge separation was first observed with series of porphyrin-quinone and porphyrin-imide dyads, respectively. ${ }^{13,14}$ In addition, long-lived CS has been reported with $\mathrm{C}_{60}$ as an electron accepting group. ${ }^{15}$

Core-substituted naphthalenediimides (NDIs) have recently emerged as powerful elements in the design of multichromophoric systems. ${ }^{16,17}$ Whereas NDIs with hydrogen atoms on the naphthalene core have been used for a long time as electron acceptors in dyads and more sophisticated architectures, ${ }^{18-21}$ their lack of absorbance in the visible region has prevented their use as chromophores. However, substitution by one, two or up to four electron-donating groups on the naphthalene core introduces a new optical transition with charge transfer character located in the visible region and with an energy that can be fine-tuned by varying the nature and number of core substituents. ${ }^{22}$ This allows the whole visible region of the electromagnetic spectrum to be covered with a single series of chromophores that differ by a few atoms only. In parallel to the decreasing energy of the first electronic transition, a continuous increase of the energy of the frontier orbitals takes place upon increasing the number and electron donating strength of the core substituents. As a consequence, these NDIs can be combined into arrays and supramolecular 


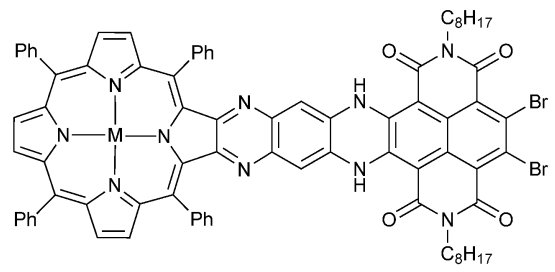

1 FbTPPNDI: $M=2 H$

2 ZnTPPNDI: $M=Z n$

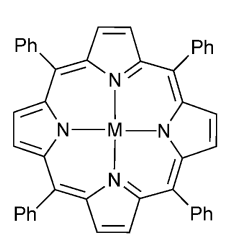

FbTPP: $\mathrm{M}=\mathbf{2 H}$ ZnTPP: $M=Z n$

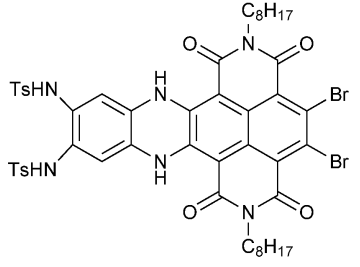

5 HBNDI
Scheme 1

architectures that do not only operate as light harvesting antennae but also undergo CS and charge conduction. These features have allowed the elaboration of highly sophisticated architectures opening original avenues for artificial photosynthesis and photovoltaics. 3,23

We present here our investigation using femtosecond-resolved spectroscopy of the excited-state properties of two dyads consisting of either a free-base or a zinc-tetraphenylporphyrin linked to a tetra core-substituted NDI unit via a rigid aromatic diaza bridge (Scheme 1). The photophysics of these compounds have been compared to those of the constituting units, namely free-base and zinc-tetraphenylporphyrin (FbTPP, ZnTPP) and a hydroquinoxaline-substituted bromo-NDI (HBNDI). A structurally relatively similar dyad composed of a zinc-chlorin and a blue NDI has recently been reported by Würthner and coworkers. ${ }^{24}$ These dyads were found to aggregate via the chlorin units and, upon optical excitation of the NDI moiety, energy transfer to the aggregated chlorins was observed to take place with a 5 ps time constant. The systems described here differ by the nature of the bridging group that introduces a strong coupling between the porphyrin and the NDI units. We will show that this leads to a delocalisation of the optical excitation over the whole molecules and that these systems should rather be considered as new chemical entities rather than as conventional donor-bridge-acceptor compounds. Moreover, the nature of the building elements is such that intramolecular CS is observed already in moderately polar solvents.

\section{Experimental section}

\section{Samples}

The synthesis of FbTPPNDI (1) and ZnTPPNDI (2) is described in detail in the ESI. $\dagger$ In brief, FbTPPNDI (1) and ZnTPPNDI (2) were synthesized by the condensation of the ortho-diamine functionalized NDI with tetraphenylporphyrin-2,3-dione in a high yield (Scheme 2). Synthesis of diamino-annulated NDIs 3 began by performing condensation of 2,3,6,7-tetrabromonaphthalene diimide $\mathbf{5}^{25}$ and<smiles>[R]N1C(=O)c2c(Br)c(Br)c3c4c(c(Br)c(Br)c(c24)C1=O)C(=O)N([R])C3=O</smiles>

$5 \mathrm{R}=-\left(\mathrm{CH}_{2}\right)-\mathrm{CH}_{3}$<smiles></smiles>

i)<smiles>Nc1cc(N)c(N=S)cc1N</smiles><smiles>[Y]#CC</smiles><smiles></smiles>

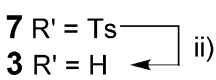

iii)<smiles>[R]C(C)=[W]</smiles>

Reaction conditions: i) $\mathrm{DMF}, 135^{\circ} \mathrm{C}, 30 \mathrm{~min}$ ii) $\mathrm{H}_{2} \mathrm{SO}_{4}$, rt. overnight.

iii) DCM, pyridine, reflux, $2 \mathrm{hrs}$

Scheme 2

1,2-diamino-4,5-bis ( $p$-toluenesulfonamido) benzene precursor $6^{26-28}$ under argon atmosphere in dry DMF at $135{ }^{\circ} \mathrm{C}$, giving green crystalline solid of 7 (HBNDI) in $87 \%$ yields. ${ }^{29}$ Deprotection of the tosyl protecting group with sulfuric acid gave $\mathbf{3}$ in $83 \%$ as a green solid. Tetraphenylporphyrin-2,3-dione 4 was prepared from tetraphenylporphyrin applying the literature known methods and obtained $25 \%$ in four steps. ${ }^{30,31}$ FbTPPNDI was achieved by the condensation of the ortho-diamine NDI 3 with 4 in DCM/pyridine at $45^{\circ} \mathrm{C}$ yielding $\mathbf{1}$ in $89 \%$ yield as a purple solid. Finally ZnTPPNDI 2 was achieved in $96 \%$ yield by metallation of 1 with using $\mathrm{Zn}(\mathrm{OAc})_{2}$ in $\mathrm{CHCl}_{3} / \mathrm{MeOH}$ at room temperature.

The solvents, toluene, dichloromethane (DCM) and benzonitrile $(\mathrm{BCN})$ were of the highest commercially available purity and were used as received.

\section{Steady-state measurements}

Absorption spectra were recorded on a Cary 50 spectrophotometer, whereas fluorescence and excitation spectra were 
measured on a Cary Eclipse fluorimeter. All fluorescence spectra were corrected for the wavelength-dependent sensitivity of the detection. For fluorescence measurements, the absorbance of the solutions at the band maximum was around 0.1 over $1 \mathrm{~cm}$. The fluorescence quantum yields were measured using cresyl violet perchlorate in ethanol $\left(\Phi_{\mathrm{fl}}=0.59\right)^{32}$ as a standard.

\section{Time-resolved fluorescence}

Fluorescence decay measurements were performed using the same time-correlated single photon counting (TCSPC) setup as described in ref. 33. Excitation was carried out with $<90$ ps pulses generated with a laser diode at $395 \mathrm{~nm}$ (PicoQuant model LDH-PC-400B) and fluorescence was detected at magic angle. The full width at half-maximum (FWHM) of the instrument response function (IRF) was around 200 ps.

\section{Transient absorption (TA)}

The experimental setup has been described in detail earlier. ${ }^{34,35}$ Excitation was performed at $400 \mathrm{~nm}$ with the frequencydoubled output of a standard $1 \mathrm{kHz}$ amplified Ti: Sapphire system (Spectra-Physics) and at $615 \mathrm{~nm}$ with a home-built two-stage non-collinear optical parametric amplifier. The pump intensity on the sample was $c a$. $1-2 \mathrm{~mJ} \mathrm{~cm}^{-2}$. The polarisation of the probe pulses was at magic angle relative to that of the pump pulses. All spectra were corrected for the chirp of the white-light probe pulses. The FWHM of the response function was $c a .150 \mathrm{fs}$. The sample solutions were placed in a $1 \mathrm{~mm}$ thick quartz cell and were continuously stirred by $\mathrm{N}_{2}$ bubbling. Their absorbance at the excitation wavelength was around 0.3 .

\section{Computational methods}

Ground-state gas-phase geometry optimisation was performed at the density functional level of theory (DFT) using the B3LYP functional, ${ }^{36}$ and a [3s2pld] basis set. ${ }^{37}$ Electronic vertical excitation energies were computed with time-dependent density functional theory (TD-DFT) using the same functional and basis set. ${ }^{38}$ The calculations were carried out using Turbomole version $6.0 .^{39}$

\section{Results}

\section{Steady-state spectroscopy}

The absorption spectra of FbTPPNDI and ZnTPPNDI are depicted in Fig. 1 together with those of the parent compounds, FbTPP, ZnTPP and HBNDI, whereas the band maxima are listed in Table $\mathrm{S} 1$ (ESI $\dagger$ ). The lowest absorption band of HBNDI with the maximum at $609 \mathrm{~nm}$ and a $\sim 1350 \mathrm{~cm}^{-1}$ vibrational progression can be ascribed to a transition associated with a substantial redistribution of the electronic density from the core substituents to the carbonyl groups. The next band at $434 \mathrm{~nm}$, corresponding most probably to the $S_{2} \leftarrow S_{0}$ transition, can be assigned to a $\pi-\pi^{*}$ transition involving mainly the NDI centre. ${ }^{40}$

The absorption spectra of the porphyrins are well known, ${ }^{41,42}$ and consist of the Q-bands in the 500-670 $\mathrm{nm}$ region and of the intense Soret band with maxima at 417 and $423 \mathrm{~nm}$ for FbTPP and ZnTPP, respectively. Although the main

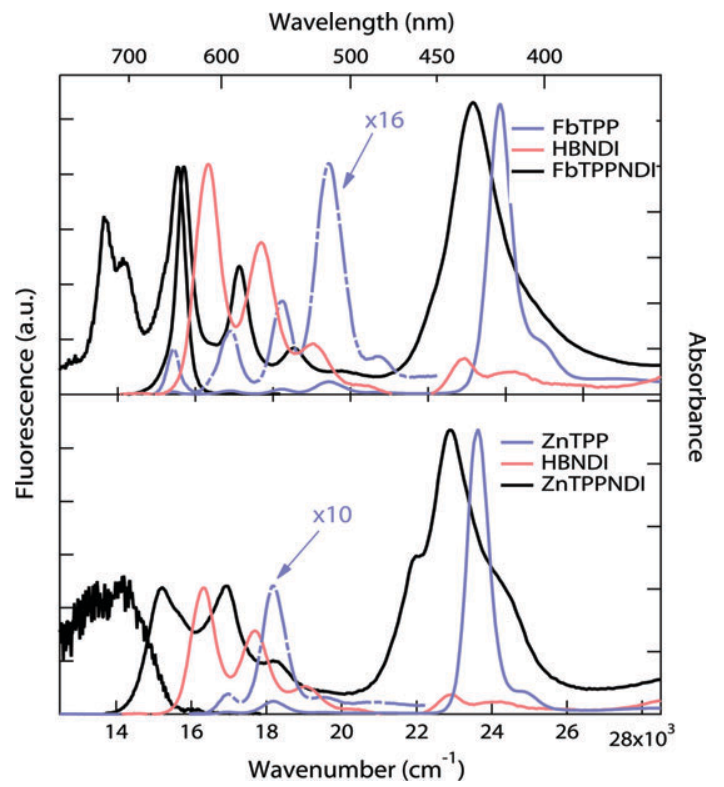

Fig. 1 Stationary absorption and fluorescence spectra of FbTPPNDI (top) and ZnTPPNDI (bottom) in toluene together with the absorption spectra of the individual units (the intensity of the $\mathrm{Q}$ band region of the porphyrins has been enlarged).

absorption features of the individual chromophores can be recognized in the absorption spectra of FbTPPNDI and ZnTPPNDI, the latter are not the composites of the individual spectra. The spectra of the dyads consist in two bands, a structured one between 500 and $700 \mathrm{~nm}$, which looks like the low energy band of HBNDI, and the other around $430 \mathrm{~nm}$, which bears a resemblance with the Soret band of the porphyrin constituent. However, the low energy band is red shifted by $724 \mathrm{~cm}^{-1}$ in FbTPPNDI and by $1110 \mathrm{~cm}^{-1}$ in ZnTPPNDI. Moreover, the energy of the vibrational progression amounts to $1400 \mathrm{~cm}^{-1}$ for FbTPPNDI, whereas for ZnTPPNDI, the energy difference between the maxima is not constant but is equal to $\sim 1600 \mathrm{~cm}^{-1}$ and $1370 \mathrm{~cm}^{-1}$, starting from the lowest energy band. The vibrational structure is also shallower than in HBNDI and in FbTPPNDI. The band around $430 \mathrm{~nm}$ is red shifted by $725 \mathrm{~cm}^{-1}$ and $546 \mathrm{~cm}^{-1}$ for FbTPPNDI and ZnTPPNDI, respectively, and substantially broadened compared to the Soret band.

Neither the Q bands of the porphyrins nor the $434 \mathrm{~nm}$ band of the NDI moiety can be discerned in the absorption spectra of the dyads. This can be explained by their weakness and by their overlap with the more intense bands of the other chromophoric moiety. However, the substantial difference between the spectrum of the dyads and those of the individual constituents points to a strong coupling between the porphyrin and the NDI units and thus to a delocalization of the excitation over the whole dyads. This leads to mixing of the NDI and porphyrin transitions. Mixing of the Q-bands of ZnTPP and of the NDI bands might explain for example the shape of the low energy band in ZnTPPNDI. The high delocalisation of the excitation is strongly supported by TD-DFT calculations performed with FbTPPNDI that show that the frontier molecular orbitals are not localized on a single chromophoric unit but extend over the whole molecule, as illustrated in 

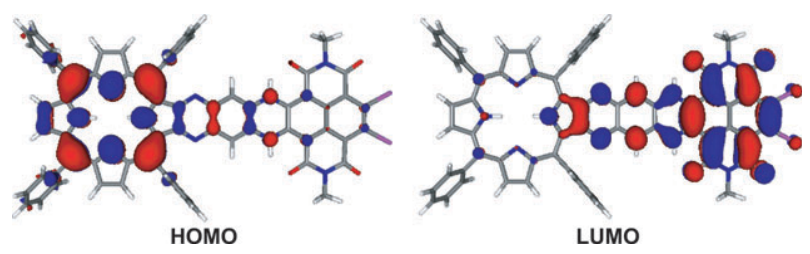

Fig. 2 Frontier molecular orbitals of FbTPPNDI drawn at the 0.02 a.u. level.

Fig. 2. These gas-phase calculations predict that the first electronic transition is at $675 \mathrm{~nm}$ with an oscillator strength of 0.2 and that it is related to a one-electron HOMO-LUMO transition. Fig. 2 shows that such a transition involves a substantial charge transfer from the FbTPP to the NDI moiety. The calculated transition energy of $1.83 \mathrm{eV}$ is in good agreement with that $1.94 \mathrm{eV}$ measured in toluene and DCM. The calculations also predict transitions at higher energies localized on either the NDI (HOMO-2-LUMO) or the FbTPP $(\mathrm{HOMO}-1-\mathrm{LUMO}+2)$ units. In the following, the two excited states populated upon excitation in the low energy band and in the $430 \mathrm{~nm}$ band will be called OPS1 (optically populated state 1) and OPS2, respectively. As will be shown below, these states are not the first two singlet excited states in polar solvents.

The absorption spectra of both dyads were found to vary markedly with increasing concentration as illustrated for FbTPPNDI in Fig. 3. As concentration becomes higher, the $430 \mathrm{~nm}$ band broadens and shifts slightly to the red, whereas shoulders appear on the low-energy side of the bands in the 550-700 nm region. A concentration dependence was also observed with ZnTPPNDI: the $430 \mathrm{~nm}$ band broadens and shifts to longer wavelength as well. However, no new shoulder appears in the 550-700 $\mathrm{nm}$ region but the vibronic structure becomes less marked and a substantial broadening of the red edge of the band appears. These effects can undoubtedly be ascribed to the formation of aggregates. The structure of these aggregates cannot be inferred from the present data. As the typical features of $\mathrm{H}$ or $\mathbf{J}$ aggregates cannot be recognized here, the aggregated dyads cannot simply be interpreted as one of these two forms. However, the presence of the twisted phenyl substituents on the porphyrin units should prevent both porphyrin-NDI and porphyrin-porphyrin stacking, as

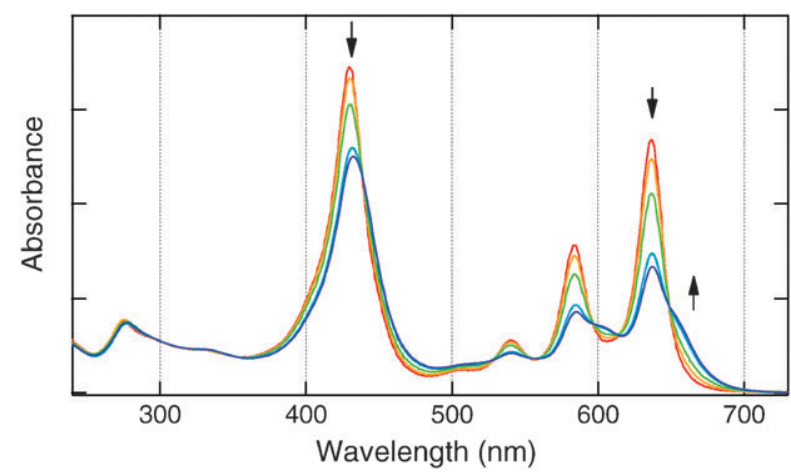

Fig. 3 Effect of increasing concentration (arrows) on the absorption spectrum of FbTPPNDI in DCM (the spectra were intensity normalized in the $300 \mathrm{~nm}$ region). already reported for FbTPP. ${ }^{43}$ Therefore, aggregation should rather take place between NDIs.

The fluorescence spectra of the dyads in toluene are depicted in Fig. 1, and the band maxima are listed in Table S1 (ESI $\dagger$ ). The emission spectrum of FbTPPNDI consists in a narrow band at $643 \mathrm{~nm}$, which is only $145 \mathrm{~cm}^{-1}$ Stokes shifted relative to the absorption band, and of a doubly peaked band (705 and $731 \mathrm{~nm}$ ). Whereas the $705 \mathrm{~nm}$ peak can be considered as the mirror image of the $584 \mathrm{~nm}$ absorption band, the $731 \mathrm{~nm}$ maximum has no equivalent in the absorption spectrum. This band cannot arise from FbTPP present as impurity because the latter fluoresces at 653 and $720 \mathrm{~nm}$. Moreover, this fluorescence spectrum is independent of the excitation wavelength and the fluorescence excitation spectra recorded at different emission wavelengths match very well the absorption spectrum. The origin of this breaking of mirror image symmetry is not clear but might indicate some structural change between the Franck-Condon and the relaxed excited state, or some differences in the shape of the potential energy surface of the excited state. The fluorescence quantum yield of FbTPPNDI in toluene amounts to 0.08 . On the other hand, the fluorescence spectrum of ZnTPPNDI in toluene comprises a broad band culminating at $700 \mathrm{~nm}\left(960 \mathrm{~cm}^{-1}\right.$ Stokes shift $)$ with a shoulder at about $750 \mathrm{~nm}$ and with a quantum yield of 0.02 .

The fluorescence quantum yield of both dyads in DCM is much smaller and could not be determined because of the presence of traces of emitting impurities.

\section{Time-resolved spectroscopy}

The fluorescence lifetimes of the dyads and of the individual chromophores have been measured by TCSPC and the results are summarized in Table 1. The fluorescence lifetimes obtained with FbTPP and ZnTPP in toluene are in good agreement with those already published. ${ }^{10}$ On the other hand, the fluorescence lifetime of HBNDI has never been reported so far and amounts to $2.7 \mathrm{~ns}$ in both toluene and DCM. The decay of the fluorescence intensity of both dyads in toluene could be well reproduced by an exponential function with a $2.7 \mathrm{~ns}$ and a 0.57 ns lifetime for FbTPPNDI and ZnTPPNDI, respectively.

TA measurements have first been performed with the individual chromophores, i.e. with FbTPP, ZnTPP and HBNDI. The TA spectra obtained with FbTPP in toluene

Table 1 Time constants obtained from a global analysis of the transient absorption spectra, $\tau_{i}$, and from TCSPC measurements, $\tau_{\mathrm{fl}}$. The lifetimes in bold are assigned to CS, those in italic to CR and those underlined to internal conversion of excited aggregates

\begin{tabular}{lllllllr}
\hline Molecule & $\lambda_{\mathrm{s}} / \mathrm{nm}$ & Solvent & $\tau_{1} / \mathrm{ps}$ & $\tau_{2} / \mathrm{ps}$ & $\tau_{3} / \mathrm{ns}$ & $\tau_{4} / \mathrm{ns}$ & $\tau_{\mathrm{ff}} / \mathrm{ns}$ \\
\hline FbTPP & 400 & Toluene & & & & & 11.0 \\
ZnTPP & 400 & Toluene & 1.3 & 29 & $\sim 2$ & $>10$ & 1.8 \\
HBNDI & 615 & Toluene & 2.2 & 42 & $\sim 2.3$ & & 2.7 \\
HBNDI/DMA & 615 & DCM & 1.25 & $\mathbf{8 . 5}$ & 0.038 & & \\
FbTPPNDI & 400 & Toluene & $\sim 0.04$ & 5.0 & $\underline{0} . \underline{6} \underline{7}$ & $>10$ & 2.7 \\
FbTPPNDI & 615 & Toluene & 0.44 & 5.0 & $\underline{\underline{6}} . \underline{7}$ & $>10$ & \\
FbTPPNDI & 400 & DCM & 3.6 & 16 & $\underline{0} . \underline{2} \underline{\underline{z}}$ & $>10$ & \\
FbTPPNDI & 615 & DCM & 2.9 & 18 & $\underline{0} . \underline{5}$ & $>10$ & \\
FbTPPNDI & 400 & BCN & 17.8 & 32 & & & \\
ZnTPPNDI & 615 & Toluene & 0.6 & 12 & 0.44 & $>10$ & 0.57 \\
ZnTPPNDI & 615 & DCM & $\mathbf{1 . 2}$ & $4.6,8.1$ & $\underline{0} . \underline{10} \underline{5}$ & $>10$ & \\
\hline
\end{tabular}


upon excitation in the Soret band $(400 \mathrm{~nm})$ and in the $Q_{x}(1,0)$ band $(615 \mathrm{~nm})$ are shown in Fig. S3 (ESI $\dagger$ ) and are in full agreement with those previously published in the literature. ${ }^{44}$ These spectra are essentially the same at both excitation wavelengths and consist in an intense negative band at $419 \mathrm{~nm}$ due to the bleach of the Soret band and of a broad positive band going from $430 \mathrm{~nm}$ up to $700 \mathrm{~nm}$ and modulated by the bleach of the $\mathrm{Q}$ bands, and by the stimulated emission. Apart from some minor spectral dynamics observed at early times, the intensity and shape of the TA spectra were found to be essentially independent of time in the 0-2 ns time window of the measurements, in agreement with the long-lived fluorescence lifetime of FbTPP. The decay of the $S_{1}$ state of FbTPP is essentially due to intersystem-crossing (ISC) to the $\mathrm{T}_{1}$ state, but this process is too slow to observe any significant spectral feature of the $T_{1}$ state within the time window of the experiment. Similar results were obtained in DCM and in $\mathrm{BCN}$.

The TA spectra recorded with ZnTPP in toluene at various time delays after $400 \mathrm{~nm}$ excitation are illustrated in Fig. S4 (ESI $\dagger$ ). Although these spectra do not differ largely from those measured with FbTPP, some spectral changes are observed in the time window of the measurements. The temporal evolution of the TA spectra was analyzed globally using the sum of four exponential functions with $1.3 \mathrm{ps}, 29 \mathrm{ps}, 2 \mathrm{~ns}$ and $>10 \mathrm{~ns}$ time constants and with the decay-associated spectra shown in Fig. S4 (ESI $\dagger$ ). The 1.3 ps spectrum exhibits a sharp negative band at $430 \mathrm{~nm}$ that can be assigned to the $\mathrm{S}_{2} \rightarrow \mathrm{S}_{0}$ stimulated emission. Thus, this time constant can be ascribed to internal conversion from $S_{2}$ to $S_{1}$. This value is in excellent agreement with that of $1.4 \mathrm{ps}$ reported for the $\mathrm{S}_{2}$ fluorescence lifetime of ZnTPP in toluene and benzene. ${ }^{45-47}$ The spectrum associated with the 29 ps lifetime has a small amplitude and corresponds to spectral dynamics that can be related to the vibrational relaxation of the $\mathrm{S}_{1}$ state. ${ }^{47}$ The $2 \mathrm{~ns}$ time constant is very close to the measured fluorescence lifetime of ZnTPP (Table 1) and can thus be safely ascribed to the decay of the $S_{1}$ state population, mainly upon ISC. The resulting $T_{1}$ state population is responsible for the residual TA signal and decays on a timescale that is much longer than the time window of the experiment.

The TA spectra recorded with HBNDI in toluene at various time delays after $615 \mathrm{~nm}$ excitation are shown in Fig. 4 together with the decay-associated spectra resulting from a global multiexponential analysis. These spectra are dominated by a structured negative band between $500 \mathrm{~nm}$ and $650 \mathrm{~nm}$ and additionally contain a weak and broad positive band below $500 \mathrm{~nm}$ and a more intense one above $650 \mathrm{~nm}$. The temporal spectral evolution could be well reproduced using the sum of three exponential functions with $2.2 \mathrm{ps}, 42 \mathrm{ps}$ and $\sim 2.3 \mathrm{~ns}$ time constants (Table 1). Considering the uncertainty associated with the limited time window of the experiment, the longest time constant is identical to the measured fluorescence lifetime. Thus the positive spectral features can be ascribed to the $S_{1}$ excited-state absorption, whereas the negative band is due to the bleach of the $\mathrm{S}_{1} \leftarrow \mathrm{S}_{0}$ transition. The spectra associated with the two shorter time constants have very weak amplitude and their 'dispersive' shape suggests that they are related to vibration and/or solvent relaxation processes. Very similar TA

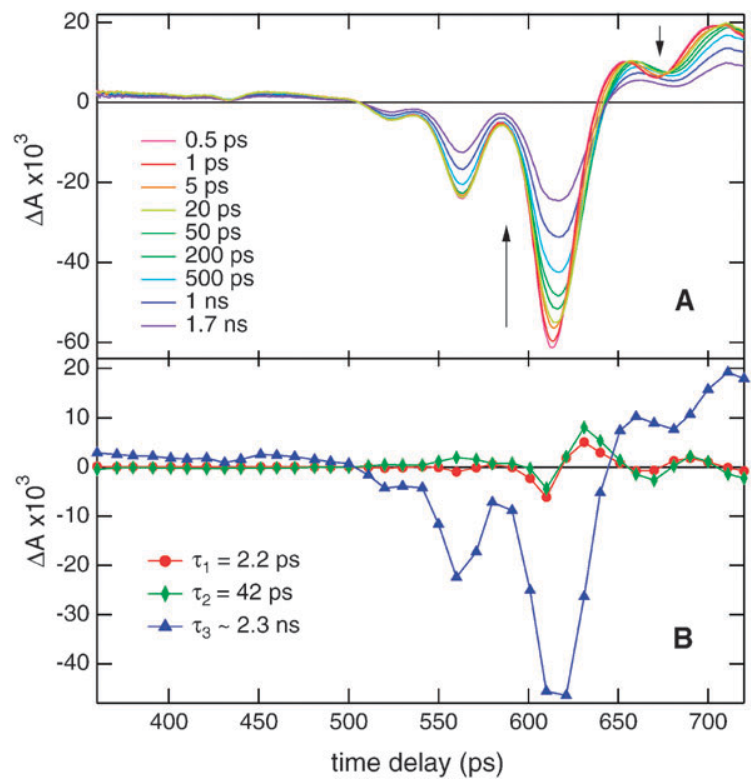

Fig. 4 (A) TA spectra recorded with HBNDI in toluene at various time delays after $615 \mathrm{~nm}$ excitation and (B) decay-associated spectra obtained from multiexponential global analysis.

spectra were obtained in DCM upon both $615 \mathrm{~nm}$ and $400 \mathrm{~nm}$ excitation.

In order to identify the possible occurrence of CS in the dyads, the $\mathrm{HBNDI}^{{ }^{-}}$radical anion has been generated by photoinduced electron transfer with the electron donor $N, N$-dimethylaniline (DMA). Fig. 5A illustrates the TA spectra recorded at different time delays after excitation of HBNDI in the presence of $3 \mathrm{M}$ DMA in DCM. Several differences with the TA spectra measured with HBNDI alone can be noticed. First, all TA bands decay to zero within about $500 \mathrm{ps}$ and, second, the positive TA bands between

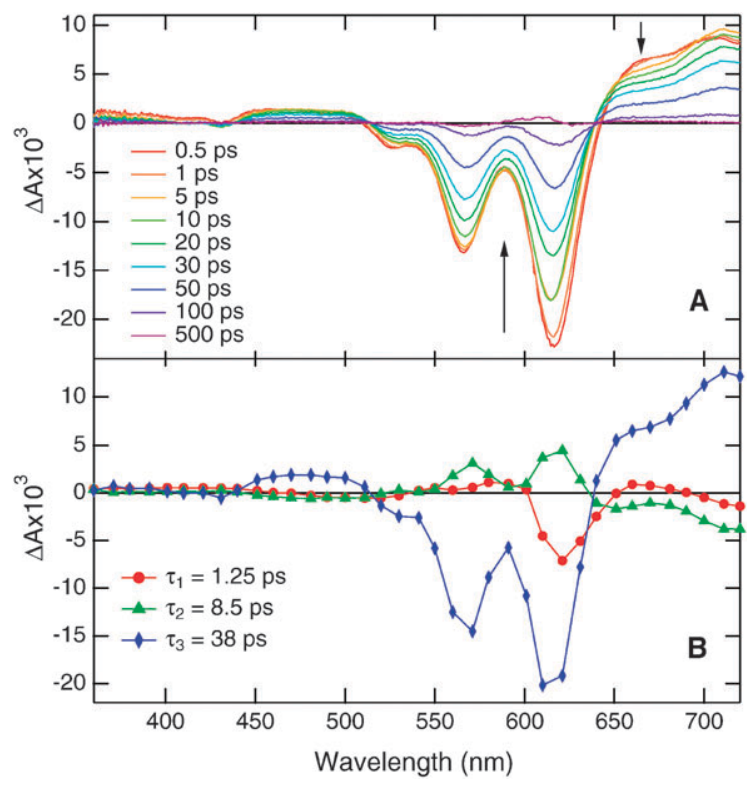

Fig. 5 (A) TA spectra recorded with HBNDI and 0.3 M DMA in DCM at various time delays after $615 \mathrm{~nm}$ excitation and (B) decayassociated spectra obtained from multiexponential global analysis. 
450 and $500 \mathrm{~nm}$ and that above $650 \mathrm{~nm}$ are more intense. The much faster decay of the spectral features can be ascribed to the occurrence of CS between HBNDI and DMA to give the $\mathrm{HBNDI}^{\bullet-} \mathrm{DMA}^{\bullet+}$ ion pair, and to the subsequent $\mathrm{CR}$ of this pair back to the neutral ground state. These spectra were also analyzed globally using the sum of three exponential functions with $1.25,8.5$ and 38 ps time constants and with the amplitude spectra shown in Fig. 5B. The 1.25 ps spectrum does not differ strongly from the $2.2 \mathrm{ps}$ spectrum obtained without the electron donor and, consequently, this time constant is most probably dominated by solvent/vibration relaxation phenomena. The 38 ps spectrum consists mainly in two positive bands and the negative and structured band due to the bleach of the $\mathrm{S}_{1} \leftarrow \mathrm{S}_{0}$ transition. This last feature indicates that this time constant can be associated with CR. Therefore, CS most probably occurs with the 8.5 ps time constant. By comparing the TA spectra obtained with HBNDI alone in DCM and with DMA, it appears that $\mathrm{HBNDI}^{\bullet-}$ mainly absorbs around $500 \mathrm{~nm}$ and $700 \mathrm{~nm}$. These absorption features are similar to those observed with the radical anion of another blue NDI. ${ }^{48}$

The TA spectra measured with FbTPPNDI in toluene upon population of OPS2 with $400 \mathrm{~nm}$ pulses are displayed in Fig. 6A. These spectra consist in several negative bands that are due to the depletion of the ground-state population. Those overlap with positive bands that extend all through the visible range. Apart from a partial decay of the bands above $500 \mathrm{~nm}$ on the $\sim 500$ ps timescale, the spectra do not exhibit strong changes within the time window of the experiment. The TA dynamics could be reproduced by global analysis using the sum of four exponential functions with $\sim 40 \mathrm{fs}, 5 \mathrm{ps}, 670 \mathrm{ps}$ and $>10$ ns time constants and with the amplitude spectra shown in Fig. 6B. The $40 \mathrm{fs}$ time constant is associated with a rise of all spectral features. As it is substantially smaller than the response function of the setup, it should be considered with caution and might be partially due to an improper correction

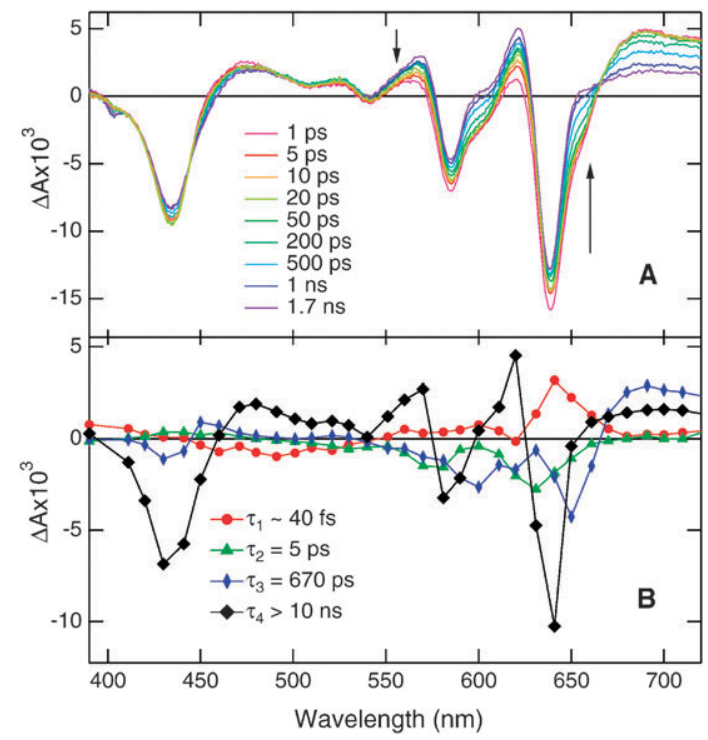

Fig. 6 (A) TA spectra recorded with FbTPPNDI in toluene at various time delays after $400 \mathrm{~nm}$ excitation and (B) decay-associated spectra obtained from multiexponential global analysis. of the cross-phase modulation. On the other hand, the $5 \mathrm{ps}$ time constant most probably originates from solvent/ vibrational relaxation as found with the individual chromophoric units. The 670 ps time constant is responsible for the spectral changes observed above $500 \mathrm{~nm}$. Its associated spectrum consists in negative bands that are slightly blue shifted with respect to the maxima of the stationary absorption spectrum. The TA spectra in the $570-650 \mathrm{~nm}$ region indicate that this $670 \mathrm{ps}$ component corresponds to the decay of shoulders at 580 and $640 \mathrm{~nm}$. As shown in Fig. 3, these shoulders can be ascribed to the absorption of FbTPPNDI aggregates present at the concentration used for the TA experiments. Finally, the last component is longer than the 2.7 ns fluorescence lifetime. In fact no such $2.7 \mathrm{~ns}$ component could be observed in the TA dynamics. Given the small fluorescence quantum yield of FbTPPNDI in toluene $\left(\Phi_{\mathrm{fl}}=0.08\right)$, this points to ISC as the main decay channel of the lowest singlet excited state, which in toluene corresponds to OPS1. Most probably the TA spectra of the $\mathrm{S}_{1}$ and $\mathrm{T}_{1}$ states do not differ enough for ISC to be observed within the limited time window of the experiment.

Similar TA measurements have been repeated upon population of OPS1 with $615 \mathrm{~nm}$ pulses. The resulting spectra are very similar to those recorded upon $400 \mathrm{~nm}$ excitation except that the spectral changes occurring on the $\sim 500$ ps timescale are more pronounced (Fig. S5, ESI $\dagger$ ). Global analysis gave the same time constants except for the shortest one, which was 0.44 ps, and almost identical decay-associated spectra. The main difference compared to $400 \mathrm{~nm}$ excitation is the amplitude of the $670 \mathrm{ps}$ associated spectrum that is twice as large. This points to a higher contribution of aggregates, in agreement with the fact that $615 \mathrm{~nm}$ coincides with an aggregate shoulder in the absorption spectrum of FbTPPNDI. To further confirm the assignment of the 670 ps time constant to aggregates, TA measurements at $615 \mathrm{~nm}$ have been repeated with a highly concentrated FbTPPNDI sample. As illustrated in Fig. S6 (ESI $\dagger)$, the early TA spectra $(<200 \mathrm{ps})$ are dominated by the bleach of the aggregate absorption. As time goes, the aggregate bleach bands decay and only those due to the bleach of the monomeric FbTPPNDI remain. Global analysis gave the same time constants as with a less concentrated sample, but the amplitude of the 670 ps associated spectrum is much larger. This clearly confirms the assignment of this time constant to the decay of the excited aggregates to the ground state. Finally, the rise of the bleach of both the $430 \mathrm{~nm}$ band and of the structured 550-650 nm band occurs within the response function of the instrument, independently of whether OPS2 or OPS1 is initially populated. This confirms that electronic excitation is delocalized over the whole molecule and that internal conversion from OPS2 down to OPS1 is ultrafast.

TA spectra were also recorded with FbTPPNDI in the more polar solvent DCM upon both $400 \mathrm{~nm}$ (Fig. 7A) and $615 \mathrm{~nm}$ excitation. As illustrated in Fig. 7A, major differences with the spectra in toluene can be observed. First, the decay of all TA bands is much faster, and their intensity decreases by a factor of about two in about 20 ps. Second, the positive TA bands present in toluene at 570 and $620 \mathrm{~nm}$, and probably due to OPS1 absorption, cannot be seen in DCM. 


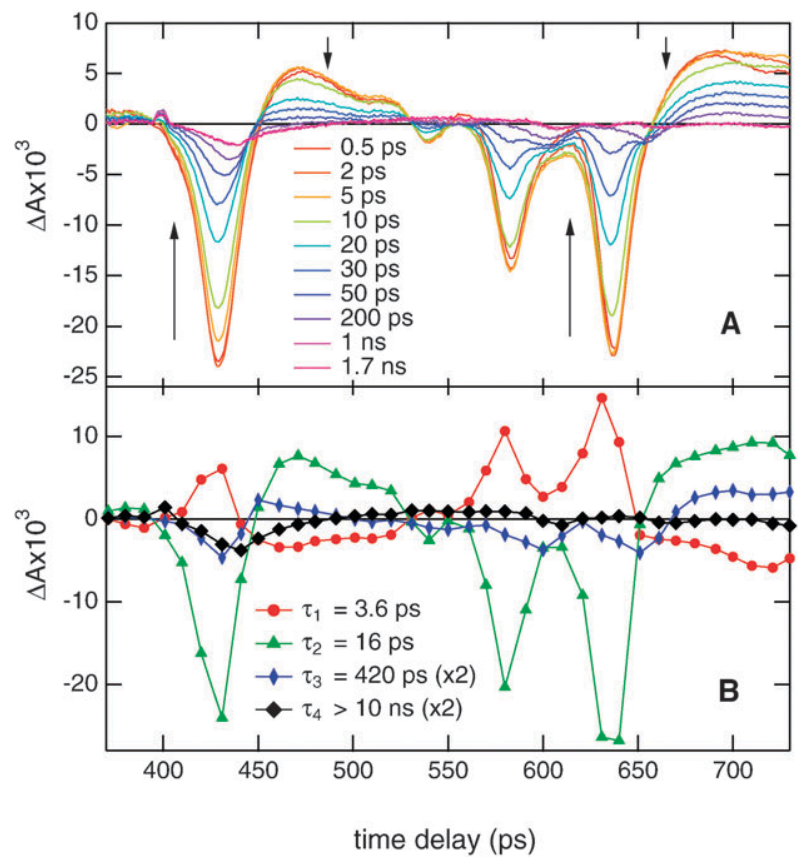

Fig. 7 (A) TA spectra recorded with FbTPPNDI in DCM at various time delays after $400 \mathrm{~nm}$ excitation and (B) decay-associated spectra obtained from multiexponential global analysis.

Global analysis of the TA data required the sum of four exponential functions with $3.6 \mathrm{ps}, 16 \mathrm{ps}, 420 \mathrm{ps}$ and $>10 \mathrm{~ns}$ time constants and with the associated spectra depicted in Fig. 7B. Most of the amplitude of the TA spectra is related to the $16 \mathrm{ps}$ time constant. The negative bands in the associated spectrum show that the largest fraction of the ground-state recovery takes place with this time constant. Moreover, the positive feature above $650 \mathrm{~nm}$ resembles that found in the spectrum of $\mathrm{HBNDI}^{\bullet-}$ (Fig. 5). Additionally, the FbTPP ${ }^{\bullet+}$ radical cation has also been reported to have an absorption maximum at $670 \mathrm{~nm} .{ }^{49} \mathrm{On}$ the other hand, this feature above $650 \mathrm{~nm}$ is present in the $3.6 \mathrm{ps}$ associated spectrum but as a negative band, indicating a rising component. This spectrum also contains two positive bands at 570 and $620 \mathrm{~nm}$ that could point to a decay of the excited-state population. Therefore, we ascribe the 3.6 ps time constant to a CS process from the lowest optically accessible excited state OPS1, and the 16 ps time constant to the decay of this charge-separated state (CSS) by CR back to the ground state.

From its associated spectrum, the $420 \mathrm{ps}$ time constant can be ascribed to the decay of the aggregate excited-state population. At the concentration used, aggregation is minor and the amplitude of this component is very small. Finally, the TA spectra do not completely decay to zero, but a very small spectrum is remaining at the longest time delay. This small residual spectrum seems be related to other aggregates.

The above assignments are supported by the TA measurements upon $615 \mathrm{~nm}$ excitation (Fig. S7, ESI $\dagger$ ) performed with a higher FbTPPNDI concentration. The early spectra are rather similar to those measured upon $400 \mathrm{~nm}$ excitation, except for the presence of pronounced shoulders in the bleach at 600 and $650 \mathrm{~nm}$ that exhibit a slower time evolution than the main bands and which are ascribed to aggregates. As time goes, the TA bands decrease rather quickly except in the 600 and $650 \mathrm{~nm}$ region, and after about $40 \mathrm{ps}$, the initial negative bands at 580 and $630 \mathrm{~nm}$ have totally vanished and the aggregate features at 600 and $650 \mathrm{~nm}$ that were initially shoulders are now distinct band maxima that decay on a $\sim 300-400$ ps timescale. A residual TA spectrum similar to that measured upon $400 \mathrm{~nm}$ excitation but with higher amplitude can be seen at the longest time delay. Global analysis of the TA dynamics yielded very similar time constants, namely $2.9,18,350 \mathrm{ps}$ and $>10 \mathrm{~ns}$, and associated spectra. The main difference was the larger amplitude of the 350 ps component due to the aggregates, in agreement with the more concentrated sample solution, and of the residual spectrum, supporting our above assignment to other aggregated forms.

Finally, TA spectra were also recorded with FbTPPNDI in an even more polar solvent, BCN, upon $400 \mathrm{~nm}$ excitation. These spectra were very similar to those in DCM except that they do not contain any significant contribution from the aggregates. As a consequence, global analysis could be performed using a biexponential function with 17.8 ps and 32 ps time constants. From the associated spectra, the shortest time constant can be ascribed to CS, whereas the longer one to $\mathrm{CR}$.

ZnTPPNDI was also investigated by TA in toluene and DCM upon 400 and $615 \mathrm{~nm}$ excitation. The TA spectra in both solvents were essentially independent of the excitation wavelength and therefore only those recorded with $615 \mathrm{~nm}$ excitation will be discussed, those in toluene being shown in Fig. 8. These spectra consist in two negative bands, one at about $430 \mathrm{~nm}$ and the other between 530 and $670 \mathrm{~nm}$, that can be assigned to the bleach of the ground-state absorption, and in three positive bands, around 390, 500 and above $690 \mathrm{~nm}$.

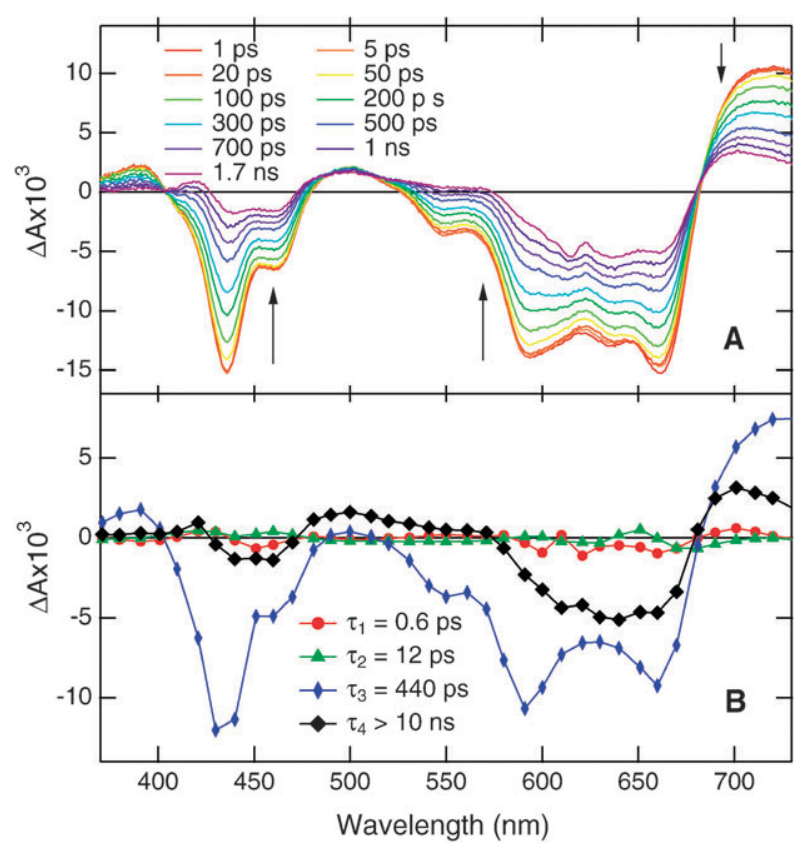

Fig. 8 (A) TA spectra recorded with ZnTPPNDI in toluene at various time delays after $615 \mathrm{~nm}$ excitation and (B) decay-associated spectra obtained from multiexponential global analysis. 
These bands decay partially on a $400 \mathrm{ps}$ timescale to a residual spectrum that is mainly characterized by the weakness of the $390 \mathrm{~nm}$ band. The TA dynamics was analyzed globally with four exponential functions with $0.6,12,443 \mathrm{ps}$ and $>10 \mathrm{~ns}$ time constants. In fact the first two components have a very weak amplitude and were only necessary to reproduce minor early spectral dynamics due most probably to solvent/ vibrational relaxation. On the other hand, the $440 \mathrm{ps}$ component is dominant and its associated spectrum points to a process that leads to a recovery of the ground-state population. Furthermore, given the error on this large time constant, it can be considered to match well the 570 ps fluorescence lifetime recorded by TCSPC. Consequently, this time constant can be ascribed to the decay of OPS1. The origin of the residual spectrum is not fully clear. A contribution from aggregates can in principle be ruled out, as both the shape and the relative amplitude of this spectrum were the same with 400 and $615 \mathrm{~nm}$ excitation, although the aggregates have a higher relative absorbance at $615 \mathrm{~nm}$. Consequently, this residual spectrum can be due to either the triplet-excited state of ZnTPPNDI or to a CSS. This point will be discussed in more detail below.

Finally, TA spectra collected with ZnTPPNDI in DCM upon $615 \mathrm{~nm}$ excitation are depicted in Fig. 9A. The spectral shape is qualitatively the same as in toluene but the dynamics is different. Global analysis of the TA dynamics required not less than the sum of five exponential functions with 1.2, 4.6, $8.1,105 \mathrm{ps}$ and $>10 \mathrm{~ns}$ time constants and with the associated spectra shown in Fig. 9B. In order to simplify the interpretation, the amplitudes associated with the 4.6 and $8.1 \mathrm{ps}$ time constants have been added. Because of their similar value and amplitude spectra, these constants will be considered together in the following. From its associated spectrum, it appears that this $\sim 6$ ps lifetime is responsible for the recovery of most of the ground-state population. Additionally, it is related to a decay of the positive TA bands at $390,490 \mathrm{~nm}$ and

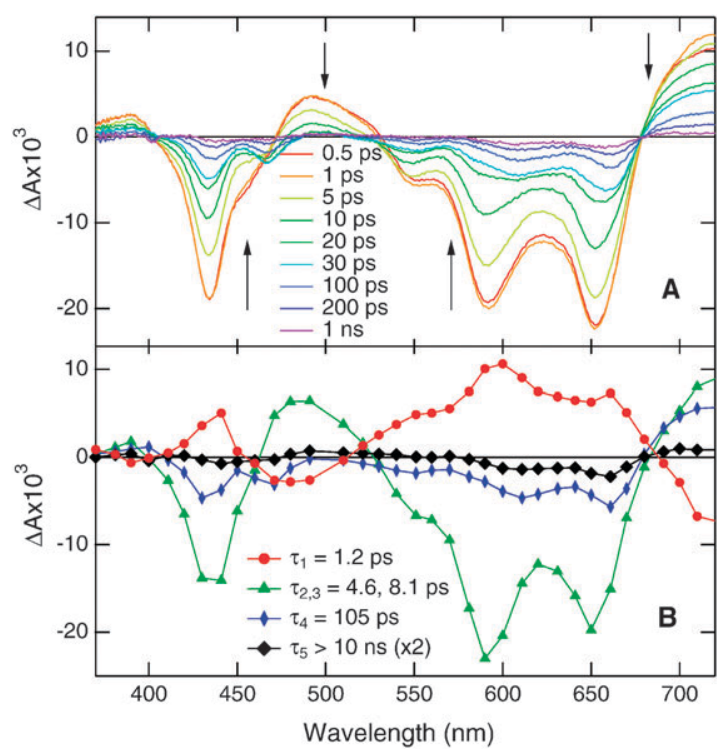

Fig. 9 (A) TA spectra recorded with ZnTPPNDI in DCM at various time delays after $615 \mathrm{~nm}$ excitation and (B) decay-associated spectra obtained from multiexponential global analysis. above $670 \mathrm{~nm}$. Given that $\mathrm{HBNDI}^{\bullet-}$ absorbs above $650 \mathrm{~nm}$ and that $\mathrm{ZnTPP}^{\bullet}{ }^{+}$has been shown to absorb around 400, 450 and $650 \mathrm{~nm},{ }^{50}$ we ascribe this $\sim 6 \mathrm{ps}$ time constant to the decay of a CSS by CR back to the neutral ground state. As the $1.2 \mathrm{ps}$ spectrum points to rises of the bands assigned to the CSS, this time constant can be ascribed to CS from OPS1. The 105 ps spectrum is dominated by a negative band in the $550-670 \mathrm{~nm}$ region that is less structured than that in the stationary absorption spectrum of ZnTPPNDI at low concentration. Furthermore, the amplitude of this 105 ps spectrum is larger upon $615 \mathrm{~nm}$ excitation, where the aggregates absorb relatively more, than upon $400 \mathrm{~nm}$ excitation. This lifetime is thus assigned to the non-radiative deactivation of the aggregates. Finally, the residual spectrum has a very small amplitude at both $400 \mathrm{~nm}$ and $615 \mathrm{~nm}$ excitation and its shape is similar to that measured in toluene. Consequently, we propose that it is due to the same species that is responsible for the large residual spectrum in toluene.

\section{Discussion}

All the above results, together with the quantum chemistry calculations, reveal that, in both FbTPPNDI and ZnTPPNDI, the porphyrin and the NDI moieties are strongly coupled, and that these species should be considered as single molecular entities with new properties rather than systems consisting in two individual units.

The most spectacular property of these molecules is probably the strong dependence of their excited-state dynamics on solvent polarity. Indeed, in non-polar solvents, the lowest singlet excited state is OPS1 that can be directly populated at $615 \mathrm{~nm}$, or indirectly upon $400 \mathrm{~nm}$ excitation and ultrafast internal conversion from OPS2. On the other hand, in the moderately polar DCM $\left(\varepsilon_{\mathrm{S}}=8.9\right)$, the emitting state has a lifetime of a few picoseconds due to the occurrence of CS. Thus in polar solvents, the lowest singlet excited state is a CSS. The energy of this state in toluene and DCM can be estimated from the following equation: ${ }^{51}$

$$
E_{\mathrm{CSS}}=E_{\mathrm{ox}}(D)-E_{\mathrm{red}}(A)+C+S
$$

where $E_{\mathrm{ox}}(D)$ and $E_{\mathrm{red}}(A)$ are the oxidation and reduction potentials of the electron donating and accepting moieties, respectively, $C$ is a correction term that accounts for the coulombic interaction between the charges separated by a distance $d_{\mathrm{DA}}$ and screened by the solvent with a static dielectric constant $\varepsilon_{\mathrm{s}}$ :

$$
C=-\frac{e^{2}}{4 \pi \varepsilon_{0} \varepsilon_{\mathrm{s}} d_{\mathrm{DA}}}
$$

and $S$ is another correction factor that accounts for the energy of the CSS in a solvent with a different dielectric constant than that used for the measurements of the redox potentials:

$$
S=-\frac{e^{2}}{8 \pi \varepsilon_{0}}\left(\frac{1}{r_{\mathrm{D}}}+\frac{1}{r_{\mathrm{A}}}\right)\left(\frac{1}{\varepsilon_{\mathrm{s}, \mathrm{pol}}}-\frac{1}{\varepsilon_{\mathrm{s}}}\right)
$$

where $r_{\mathrm{A}}$ and $r_{\mathrm{D}}$ are the radii of the electron donating and accepting units, and $\varepsilon_{\mathrm{s}, \mathrm{pol}}$ is the dielectric constant of the polar solvent used for the determination of the redox potentials. 
Table 2 Energy of the charge-separated states, $E_{\mathrm{CSS}}$, and driving force for photoinduced charge separation ${ }^{a}, \Delta G_{\mathrm{CS}}$, calculated with eqn (1)

\begin{tabular}{llll}
\hline CSS & Solvent & $E_{\mathrm{CSS}} / \mathrm{eV}$ & $\Delta G_{\mathrm{CS}} / \mathrm{eV}$ \\
\hline FbTPP $^{\cdot+}$ NDI $^{-}-$ & Toluene & 2.7 & +0.76 \\
FbTPP $^{+}$NDI $^{-}$ & DCM & 2.0 & +0.06 \\
FbTPP $^{-}$NDI $^{+}$ & Toluene & 3.2 & +1.26 \\
FbTPP $^{+}$NDI $^{-}$ & DCM & 2.5 & +0.56 \\
ZnTPP $^{-}$NDI $^{-}$ & Toluene & 2.5 & +0.65 \\
ZnTPP $^{+}{ }^{+}$NDI $^{-}$ & DCM & 1.8 & -0.05 \\
ZnTPP $^{-}$NDI $^{+}$ & Toluene & 3.4 & +1.55 \\
ZnTPP $^{-}{ }^{+}$NDI $^{-}$ & DCM & 2.7 & +0.85
\end{tabular}

${ }^{a}$ Calculated assuming $E_{\text {red }}(\mathrm{HBNDI})=-0.81 \mathrm{~V}, E_{\mathrm{ox}}(\mathrm{HBNDI})=$ $1.14 \mathrm{~V}$, with $E_{\text {red }}($ FbTPP $)=-1.21 \mathrm{~V}, E_{\text {ox }}($ FbTPP $)=1.08 \mathrm{~V}$, $E_{\text {red }}(\mathrm{ZnTPP})=-1.42 \mathrm{~V}, E_{\mathrm{ox}}(\mathrm{ZnTPP})=0.82 \mathrm{~V}$ (all potentials are vs. SCE, those for the porphyrins are taken from ref. 52), and with $E_{\mathrm{OPS} 1}(\mathrm{FbTPPNDI})=1.94 \mathrm{eV}$ and $E_{\mathrm{OPS} 1}(\mathrm{ZnTPPNDI})=1.85 \mathrm{eV}$.

Usually, this is a highly polar solvent like acetonitrile or DMF and thus $\varepsilon_{\mathrm{s}, \mathrm{pol}}>30$.

The electrochemistry of FbTPPNDI and ZnTPPNDI is not known. However, in order to have an estimate of the nature and energy of the CSS, the redox potentials of the individual units can be used. Those of FbTPP and ZnTPP have been reported in literature, ${ }^{52}$ whereas those of HBNDI are unknown. However, as the redox properties of the core-substituted NDIs have been shown to be correlated with their $\mathrm{S}_{1} \leftarrow \mathrm{S}_{0}$ energy gap, the redox potentials of HBNDI can be expected to be close to those of a blue NDI with two amino core substituents and with similar $S_{1} \leftarrow S_{0}$ gap (compound 17 in ref. 17). Therefore the redox values of this compound, namely $E_{\text {red }}=-0.81 \mathrm{~V} v s . \mathrm{SCE}$ and $E_{\mathrm{ox}}=1.14 \mathrm{~V} v s . \mathrm{SCE},{ }^{53}$ have been used for those of HBNDI. These values indicate that the NDI unit can in principle act both as an electron acceptor and donor, the same being true for the porphyrins. In order to establish the direction of the CS, the energy of the CSS for both porphyrin to NDI and NDI to porphyrin CS has been estimated using eqn (1), and the resulting values are listed in Table 2. For these calculations, the radii of the porphyrin and of the NDI were taken as 5.7 and $4.0 \AA$, respectively, and a $d_{\text {DA }}$ value of $13.9 \AA$, corresponding to the centre-to-centre distance between the porphyrin and NDI units, was used. Although these $E_{\mathrm{CSS}}$ values should only be considered as estimates, they give precious information on the possible occurrence of CS and on its direction. From Table 2, it clearly appears that the porphyrin to NDI CS is energetically more favourable by about $0.4 \mathrm{eV}$ and $0.9 \mathrm{eV}$ in FbTPPNDI and ZnTPPNDI, respectively, than CS in the opposite direction. Fig. 10 shows the energy level schemes of these two molecules in toluene and DCM. The energy of the emitting state has been determined from the stationary absorption and emission spectra. This scheme shows that, in toluene, the lowest singlet excited state is clearly the emissive state, OPS1. On the other hand in DCM the lowest singlet excited state of ZnTPPNDI is the $\mathrm{ZnTPP}^{\bullet}{ }^{+} \mathrm{NDI}^{\bullet}-\mathrm{CSS}$, whereas for FbTPPNDI, OPS1 and the $\mathrm{FbTPP}^{\circ} \mathrm{NDI}^{-}$- CSS are very close in energy.

The observed solvent dependence of the excited state dynamics of both compounds can now be safely assigned to the occurrence of CS from the porphyrin to the NDI unit in DCM. This CS takes place with the time constants of 3.6 and

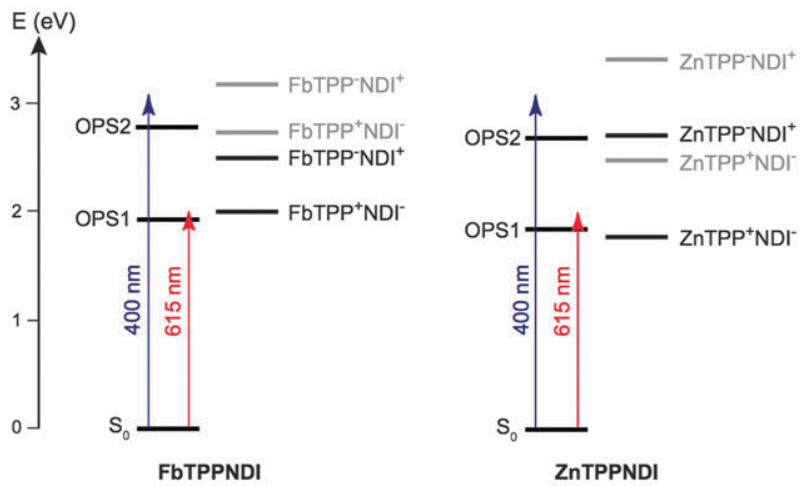

Fig. 10 Energy levels of FbTPPNDI and ZnTPPNDI in DCM and in toluene (grey).

1.2 ps in FbTPPNDI and ZnTPPNDI, respectively. Such very fast CS suggests that the energy of the CSS is probably overestimated and that of FbTPPNDI in DCM should be lower by about $0.1-0.2 \mathrm{eV}$. Despite this, the driving force for $\mathrm{CS}, \Delta G_{\mathrm{CS}}=E_{\mathrm{CSS}}-E_{\mathrm{OPS} 1}$, is small and the fast CS is most likely due to a small intramolecular reorganization energy introduced by the rigidity of the molecules and especially to a very large electronic coupling, in agreement with the strong coupling between the porphyrin and NDI units suggested by the stationary electronic spectra. As a consequence, $\mathrm{CS}$ is most probably an adiabatic process and its dynamics should be solvent controlled. ${ }^{54}$ The solvation dynamics of DCM has been shown to be multiphasic with a longest time constant of 1 ps. ${ }^{55}$ Given the weak driving force for $\mathrm{CS}$, the whole solvation energy is needed to bring the CSS below OPS1 DCM. Therefore CS in DCM cannot be faster than the slowest solvation component. On the other hand, the CS time constant of FbTPPNDI in BCN was found to amount to $17.8 \mathrm{ps}$, i.e. substantially slower than in DCM despite the larger dielectric constant of this solvent $\left(\varepsilon_{\mathrm{s}}=26\right)$ and the more favourable driving force for $\operatorname{CS}\left(\Delta G_{\mathrm{CS}}=-0.05 \mathrm{eV}\right)$. This difference fully supports a solvent-controlled adiabatic CS as the solvation dynamics of $\mathrm{BCN}$ is considerably slower than that of DCM with time constants of 5.3 and 25 ps. ${ }^{55}$ As pointed by the quantum chemistry calculations, the first electronic transition already involves a substantial charge transfer from the porphyrin to the NDI units. Therefore, the population of the CSS is most probably associated with a higher spatial localization and decoupling of the charges induced by solvation.

On the other hand, CR is very fast as well, but markedly slower than CS. This could be accounted for by a smaller electronic coupling, as the electronic wavefunction of the CSS can be expected to differ more from that of the ground state than from that of OPS1. Moreover, the driving force for CR, $\Delta G_{\mathrm{CR}}=-E_{\mathrm{CSS}} \approx-2 \mathrm{eV}$, corresponds to the onset the inverted regime. ${ }^{56}$

Both the TA data and the energetic considerations indicate that CS is not operative in a non-polar solvent like toluene. Nevertheless, the TA measurements reveal that the population of OPS1, which is the $S_{1}$ state in this case, does not decay entirely to the ground state. As the CSS is above this state, the only possible decay channel is ISC. The energy of this state is 
not known, but as the $S_{1}$ state is predicted to have a substantial charge transfer character, the $S_{1}-T_{1}$ gap can be expected to be rather moderate and not larger than that of FbTPP and ZnTPP, which amounts to $0.5 \mathrm{eV} .^{57,58}$

The faster ISC in ZnTPPNDI compared to FbTPPNDI can be explained by the heavy atom effect introduced by the $\mathrm{Zn}$ atom, which is already responsible for the shorter fluorescence lifetime of ZnTPP compare to FbTPP. Furthermore, the faster ISC in both FbTPPNDI and ZnTPPNDI compared to that in the corresponding porphyrins could be accounted for by the heavy atom effect associated with the two $\mathrm{Br}$ atoms on the NDI unit.

Despite this, an involvement of aggregated ZnTPPNDI molecules in the residual TA spectrum measured in toluene cannot be fully excluded. Our measurements show clearly that both dyads are prone to aggregation. Clearly, the lifetime of the excited aggregate is not as solvent dependent as those of the monomeric molecules, indicating that CS most probably does not play an important role in their excited-state dynamics. When excited, these species most probably decay to the ground state by internal conversion or intraband scattering.

Finally, the residual TA spectrum measured with ZnTPPNDI in DCM has been assigned to the same species as that responsible for the residual spectrum in toluene that we just ascribed to the $T_{1}$ state. The presence of a triplet state population in DCM is surprising because CS is so much faster than ISC that the $T_{1}$ yield should essentially be zero. We suggest that this very small $T_{1}$ population is generated upon triplet CR from the CSS. This process, which has been observed in geminate ion pairs with heavy atoms, ${ }^{59}$ is possible when the CSS is located between the emitting state and its corresponding $\mathrm{T}_{1}$ state and in the presence of heavy atoms that scramble the spin state of the CSS. All these conditions are probably fulfilled here with $\mathrm{Zn}$ and $\mathrm{Br}$ atoms and with a CSS lying very close in energy to the emitting state. Unfortunately, the resulting triplet population is much too small to allow a detailed study of its dynamics.

\section{Concluding remarks}

Contrary to most dyads investigated so far, the porphyrinNDI dyads reported here are very strongly coupled and behave like new chemical entities with distinct stationary absorption and emission spectra and excited-state dynamics. The excited-state dynamics of both FbTPPNDI and ZnTPPNDI is strongly solvent dependent with a lifetime of the emissive state that shortens by a factor of 500-1000 when going from a non-polar to a medium polarity solvent. This impressive effect is due to both the strong electronic coupling between the electron donating and accepting units and to the driving force of charge separation that is largely positive in non-polar solvents and slightly favourable in polar solvents. Such weakly negative driving force, together with a very large electron coupling, suffices to make this process ultrafast. The fast charge recombination of the ensuing charge-separated state prevents the use of these molecules as such for applications in artificial photosynthesis or photovoltaics. However, if coupled to secondary donors or acceptors, these molecules could be very efficient building blocks in more sophisticated supramolecular architectures that could act in the absorption of light and initiate long lived charge separation.

\section{Acknowledgements}

This work was supported by the Fonds National Suisse de la Recherche Scientifique through Project Nr. 200020-124393 and by the University of Geneva. SVB and SJL gratefully acknowledge the Australian Research Council for support under the Discovery program (DP0878756 \& DP0878220) and SVB is an Australian Postdoctoral Fellow of the Australian Research Council. We also thank Ruth F. Oliver for her help in preparing and purifying diamino-bis( $p$-toluenesulfonamido)benzene precursor.

\section{Notes and references}

1 T. van der Boom, R. T. Hayes, Y. Zhao, P. J. Bushard, E. A. Weiss and M. R. Wasielewski, J. Am. Chem. Soc., 2002, 124, 9582-9590.

2 F. Würthner, Z. Chen, F. J. M. Hoeben, P. Osswald, C. C. You, P. Jonkheijm, J. v. Herrikhuyzen, A. P. H. J. Schenning, P. P. A. M. vanderSchoot, E. W. Meijer, E. H. A. Beckers, S. C. J. Meskers and R. A. J. Janssen, J. Am. Chem. Soc., 2004, 126, 10611-10618.

3 S. Bhosale, A. L. Bhosale, P. Talukdar, A. Fürstenberg, N. Banerji, E. Vauthey, G. Bollot, J. Mareda, C. Röger, F. Würthner, N. Sakai and S. Matile, Science, 2006, 313, 84.

4 R. Bhosale, A. Perez-Velasco, V. Ravikumar, R. S. K. Kishore, O. Kel, A. Gomez-Casado, P. Jonkheijm, J. Huskens, P. Maroni, M. Borkovec, T. Sawada, E. Vauthey, N. Sakai and S. Matile, Angew. Chem., 2009, 6461-6464.

5 R. W. Wagner, J. S. Lindsey, J. Seth, V. Palaniappan and D. F. Bocian, J. Am. Chem. Soc., 1996, 118, 3996-3997.

6 N. Aratani, A. Osuka, Y. H. Kim, D. H. Jeong and D. Kim, Angew. Chem., Int. Ed., 2000, 39, 1458.

7 O. Mongin, A. Pla-Quintana, F. Terenziani, D. Drouin, C. Le Droumaget, A.-M. Caminade, J.-P. Majoral and M. BlanchardDesce, New J. Chem., 2007, 31, 1354-1367.

8 G. Kodis, Y. Terazono, P. A. Liddell, J. Andréasson, V. Garg, M. Hambourger, T. A. Moore, A. L. Moore and D. Gust, J. Am. Chem. Soc., 2006, 128, 1818.

9 P. G. VanPatten, A. P. Shreve, J. S. Lindsey and R. J. Donohoe, J. Phys. Chem. B, 1998, 102, 4209.

10 A. Morandeira, E. Vauthey, A. Schuwey and A. Gossauer, J. Phys. Chem. A, 2004, 108, 5741-5751.

11 J. Wiberg, L. Guo, K. Petterson, D. Nilsson, T. Ljungdahl, J. Mårtensson and B. Albinsson, J. Am. Chem. Soc., 2007, 129, 155-163.

12 K. Ohkubo, P. J. Sintic, N. V. Tkachenko, H. Lemmetyinen, W. E. Z. Ou, J. Shao, K. M. Kadish, M. J. Crossley and S. Fukuzumi, Chem. Phys., 2006, 326, 3-14.

13 M. R. Wasielewski, N. P. Niemczyk, W. A. Svec and E. B. Pewitt, J. Am. Chem. Soc., 1985, 107, 1080.

14 N. Mataga, H. Chosrowjan, Y. Shibata, N. Yoshida, A. Osuka, T. Kikuzawa and T. Okada, J. Am. Chem. Soc., 2001, 123, 12422.

15 A. Lembo, P. Tagliatesta, D. M. Guldi, M. Wielopolski and M. Nuccetelli, J. Phys. Chem. A, 2009, 113, 1779-1793.

16 F. Würthner, A. Shahadat, C. Thalacker and T. Debaerdemaeker, Chem.-Eur. J., 2002, 8, 4742.

17 N. Sakai, J. Mareda, E. Vauthey and S. Matile, Chem. Commun., 2010, 46, 4225-4237.

18 S. R. Greenfield, W. A. Svec, D. Gosztola and M. R. Wasielewski, J. Am. Chem. Soc., 1996, 118, 6767-6777.

19 M. Borgstroem, N. Shaikh, O. Johansson, M. F. Anderlund, S. Styring, B. Aakermark, A. Magnuson and L. Hammarstroem, J. Am. Chem. Soc., 2005, 127, 17504-17515.

20 S. J. Langford, M. J. Latter and C. P. Woodward, Photochem. Photobiol., 2006, 82, 1530-1540.

21 I. V. Sazanovich, M. A. H. Alamiry, J. Best, R. D. Bennett, O. V. Bouganov, E. S. Davies, V. P. Grivin, A. J. H. M. Meijer, 
V. F. Plyusnin, K. L. Ronayne, A. H. Shelton, S. A. Tikhomirov, M. Towrie and J. A. Weinstein, Inorg. Chem., 2008, 47, 10432-10445. 22 S. Bhosale, C. Jani and S. Langford, Chem. Soc. Rev., 2008, 37, 331-342.

23 R. Bhosale, J. Misek, N. Sakai and S. Matile, Chem. Soc. Rev., 2010, 39, 138-149.

24 C. Röger, M. G. Müller, M. Lysetska, Y. Miloslavina, A. R. Holzwarth and F. Würthner, J. Am. Chem. Soc., 2006, 128, 6542-6543.

25 X. Gao, W. Qiu, X. Yang, Y. Liu, Y. Wang, H. Zhang, T. Qi, Y. Liu, K. Lu, C. Du, Z. Shuai, G. Yu and D. Zhu, Org. Lett., 2007, 9, 3917-3920.

26 G. W. H. Cheeseman, J. Am. Chem. Soc., 1962, 84, 1171-1176.

27 S. D. Starnes, S. Arungundram and C. H. Saunders, Tetrahedron Lett., 2002, 43, 7785-7788.

28 A. Kleineweischede and J. Mattay, Eur. J. Org. Chem., 2006, 947-957.

29 S. V. Bhosale, S. V. Bhosale, M. B. Kalyankar and S. J. Langford, Org. Lett., 2009, 11, 5418-5421.

30 V. Promarak and P. L. Burn, J. Chem. Soc., Perkin Trans. 1, 2001, 14-20.

31 M. J. Crossley, C. S. Sheehan, T. Khoury, J. R. Reimers and P. J. Sintic, New J. Chem., 2008, 32, 340-352.

32 D. Madge, J. H. Brannon, T. L. Cremers and J. Olmsted III, J. Phys. Chem., 1979, 83, 695-699.

33 I. Petkova, G. Dobrikov, N. Banerji, G. Duvanel, R. Perez, D. Dimitrov, P. Nikolov and E. Vauthey, J. Phys. Chem. A, 2010, 114, 10-20.

34 G. Duvanel, N. Banerji and E. Vauthey, J. Phys. Chem. A, 2007, 111, 5361-5369.

35 N. Banerji, G. Duvanel, A. Perez-Velasco, S. Maity, N. Sakai, S. Matile and E. Vauthey, J. Phys. Chem. A, 2009, 113, 8202-8212.

36 J. P. Perdew, Phys. Rev. B: Condens. Matter Mater. Phys., 1986, 33, 8822-8824.

37 A. Schäfer, H. Horn and R. Ahlrichs, J. Chem. Phys., 1992, 97, 2571-2577.

38 R. Bauernschmitt and R. Ahlrichs, Chem. Phys. Lett., 1996, 256, 454-464.
39 R. Ahlrichs, M. Bär and M. Häser, Chem. Phys. Lett., 1989, 162, 165-169.

40 C. Röger and F. Würthner, J. Org. Chem., 2007, 72, 8070-8075.

41 K. Kalyanasundaram, Photochemistry of polypyridine and porphyrin complexes, Academic Press, San Diego, 1992.

42 M. Gouterman, J. Mol. Spectrosc., 1961, 6, 139.

43 D. L. Akins, H.-R. Zhu and C. Guo, J. Phys. Chem., 1996, 100, $5420-5425$.

44 J. Rodriguez, C. Kirmaier and D. Holten, J. Am. Chem. Soc., 1989, 111, 6500 .

45 G. G. Gurzadyan, T.-H. Tran-Thi and T. Gustavsson, J. Chem. Phys., 1998, 108, 385-388.

46 A. Morandeira, L. Engeli and E. Vauthey, J. Phys. Chem. A, 2002, 106, 4833-4837.

47 H.-Z. Yu, J. S. Baskin and A. H. Zewail, J. Phys. Chem., 2002, 106, 9845.

48 N. Banerji, A. Fürstenberg, S. Bhosale, A. L. Sisson, N. Sakai, S. Matile and E. Vauthey, J. Phys. Chem. B, 2008, 112, 8912-8922.

49 Z. Gasyna, W. R. Browett and M. J. Stillman, Inorg. Chem., 1985, 24, 2440-2447.

50 A. N. Okhrimenko, A. V. Gusev and M. A. J. Rodgers, J. Phys. Chem. A, 2005, 109, 7653 .

51 A. Weller, Z. Phys. Chem. Neue Folge, 1982, 133, 93.

52 A. Giraudeau, H. J. Callot and M. Gross, Inorg. Chem., 1979, 18, 201-206.

53 A. L. Sisson, N. Sakai, N. Banerji, A. Fürstenberg, E. Vauthey and S. Matile, Angew. Chem., Int. Ed., 2008, 47, 3727-3729.

54 I. Rips and J. Jortner, J. Chem. Phys., 1987, 87, 2090-2104.

55 M. L. Horng, J. A. Gardecki, A. Papazyan and M. Maroncelli, J. Phys. Chem., 1995, 99, 17311-17337.

56 E. Vauthey, J. Phys. Chem. A, 2001, 105, 340.

57 M. Gouterman and G.-M. Khalil, J. Mol. Spectrosc., 1974, 53, $88-100$.

58 A. Harriman, J. Chem. Soc., Faraday Trans. 1, 1980, 76, 1978-1985.

59 O. Nicolet and E. Vauthey, J. Phys. Chem. A, 2003, 107, 5894. 